\title{
Movement Protein: A Gatekeeper for Cell-To-Cell Transportation of Plant Viruses
}

\author{
Priyanka Kumari, Jitesh Kumar, Tushar Ranjan and Ravi Ranjan Kumar*
}

Department of Molecular Biology \& Genetic Engineering, Bihar Agricultural University, Sabour, Bhagalpur-813210 (India)

*Corresponding author

\section{Keywords}

Movement Protein

(MP), Viral

movement,

Plasmodesmata,

Virion maturation

Article Info

Accepted:

10 March 2020

Available Online:

10 April 2020
A B S T R A C T 


\section{Introduction}

A movement protein (MP) is a non-structural protein which is encoded by some plant viruses to allow their movement one infected cell to neighbouring cell. It is defined as plant viruses- encoded factors that interact with plasmodesmata (Pd) to mediate the intracellular spread of virus infection (Lucas, 2006) (Table 1). Role of movement proteins in coordinating the replication of viral genomes and the vectorial movement of the progeny genomes through the infected cell, as well as into adjacent cells. MP are thus providing unique approaches to unravel the fundamental mechanisms by which macromolecular transport is directed and integrated within and between plant cells (Lazarowitz, 1999).

We now know that viruses subvert an intracellular communication network that supports the trafficking of wide spectrum of endogenous protein and ribonucleo protein complexes that play non-cell autonomous roles in development and physiological processes. Moreover, successful spread of infection appears to rely upon the ability of a virus to counteract of the plant (Hull, 1991). These MPs could increase the size exclusion limits (SEL) of plasmodesmata when expressed in transgenic plants or introduced by microinjection into leaf mesophyll cells, and it appeared that cell-to-cell spread of infection could occur in the absence of virus particles.

Based on these findings and additional data, it was suggested that complexes of viral RNA and MP move from cell to cell (Lazarowitz, 1999). MP may thus belong to a larger group of protein that facilitates the intracellular trafficking of macromolecules through a variety of cellular functions. Plant viruses revealed that $\mathrm{PD}$ allow the cell-to-cell trafficking of virally encoded proteins, termed the movement proteins (MPs). This non-cellautonomous protein (NCAP) pathway is similarly employed by the host to traffic macromolecules. Viral MPs bind RNA/DNA in a sequence nonspecific manner to form nucleoprotein complexes (NPC). Host proteins are then involved in the delivery of MPs and NPC to the PD orifice, and a role for the cytoskeleton has been implicated. The bipartite geminiviruses require two MPs, but not $\mathrm{CP}$, for cell-to-cell or systemic infection. These single-stranded DNA (ssDNA) viruses replicate through double-stranded DNA intermediates in the nucleus, and it is this nuclear aspect that necessitates two MPs (MPB and NSP [for nuclear shuttle protein, formerly BR1 or BV1]) with distinct functions to cooperatively move the viral genome (Sanderfoot and Lazarowitz, 1996).

Movement proteins modify the plasmodesmata by one of two wellunderstood molecular mechanisms. The movement proteins of many plant viruses form a transport tubule within the pore of the plasmodesmata that allows the transport of mature virus particles (Boevinkand Oparka, 2005). Examples of viruses that use this mechanism are cowpea mosaic virus (CPMV) and tomato spotted wilt virus (TSWV).

The second mechanism by which movement proteins work is by associating with and coating the genome of the virus, causing the ribonucleoprotein complexes to be transported through plasmodesmata into neighbouring cells. TMV's 30 KDa movement protein acts via this mechanism, although it may also have other roles in infection (Deom, 1987, Citovsky, 1992 and Wolf, 1989). Virusencoded MP are the principal strategy by which all plant viruses counter the primary physical defense of the plant to infection - the cell wall - to produce systemic infection and disease. These proteins act at the molecular and cellular level has increased enormously in 
the past decade and ushered in an exciting new era of plant virology as an approach to investigating plant cell structure and function. The earliest studies focused on how movement proteins interacted with plasmodesmata, and were an important element in demonstrating the dynamic nature of these intercellular channels. Current efforts are focused on the role of movement proteins in coordinating the replication of viral genomes and the vectorial movement of the progeny genomes through the infected cell, as well as into adjacent cells. Movement proteins are thus providing unique approaches to unravel the fundamental mechanisms by which macromolecular transport is directed and integrated within and between plant cells (Waigmann, 1994).

Trafficking of NCAPs through the PD structure involves three steps in which the MP

(a) Interacts with a putative PD docking complex,

(b) Induces dilation in the PD microchannels, and

(c) Binds to an internal translocation system for delivery into the neighbouring cytoplasm.

Viral genera that use this NCAP pathway have evolved a combination of a MP and ancillary proteins that work in concert to enable the formation of a stable NPC that can compete with endogenous NCAPs for the PD trafficking machinery(Cilia 2004,Haywood 2002,Sessions 2000 and Wu 2003). Incompatible MP-host protein interactions may underlie observed tissue tropisms and restricted infection domains. These pivotal discoveries are discussed in terms of the need to develop a more comprehensive understanding of the (a) three-dimensional structure of MPs, (b) PD supramolecular complex, and (c) host proteins involved in this cell-to-cell trafficking process.
The continued investigation of the function of MPs, encoded by a number of very different viruses, from the perspective of what the virus must accomplish-namely, to coordinate replication of the virus genome and the vectorial movement of the progeny genomes from the infected cell into adjacent cells - has expanded this focus to include using MPs to unravel the fundamental mechanisms by which macromolecular transport is directed and integrated within and between plant cells.

MPs not only use plasmodesmata but they can modify and at times can even intercept these intercellular channels, depending on whether they function in fully differentiated or developing cells. It is not merely coincidental that quite specific and distinct interactions occur between the cortical endoplasmic reticulum (ER) and the MPs encoded by TMV or the DNA-containing bipartite geminivirus squash leaf curl virus (SqLCV). These distinct interactions provide new approaches for identifying and investigating the functional importance of subdomains within the ER(Lucas 2006).

Plasmodesmata are intercellular channels that establish a symplastic communication pathway between neighbouring cells in plants. Owing to this role, opportunistic microbial pathogens have evolved to exploit plasmodesmata as gateways to spread infection from cell to cell within the plant. However, although these pathogens have acquired the capacity to breach the plasmodesmal trafficking pathway, plants are unlikely to relinquish control over a structure essential for their survival so easily. plasmodesmata play an active role in plant immunity against viral, fungal and bacterial pathogens. Pathogens differ in their lifestyles and infection modes, and present the defense strategies that plants have adopted to prevent the intercellular spread of an infection (JungYoun Lee 2011). 
Tomato bushy stunt virus and its cell-to-cell movement protein (MP; P22) provide valuable tools to study trafficking of macromolecules through plants. wild-type P22 and selected movement-defective P22 amino acid substitution mutants were equivalent for biochemical features commonly associated with MPs (i.e. RNA binding, phosphorylation, and membrane partitioning), generated the hypothesis that their movement defect was caused by improper interaction between the P22 mutants and one or more host factors.

P22 was used as bait in a yeast (Saccharomyces cerevisiae) two-hybrid screen with a tobacco (Nicotiana tabacum) cDNA library, which identified a new plant homeodomain leucine-zipper protein that reproducibly interacted with $\mathrm{P} 22$ but not with various control proteins. These results were confirmed with an independent in vitro binding test. An mRNA for the host protein was detected in plants, and its accumulation was enhanced upon Tomato bushy stunt virus infection of two plant species. The significance of this interaction was further demonstrated by the failure of the homeodomain protein to interact efficiently with two of the well-defined movementdeficient P22 mutants in yeast and in vitro (Desvoyes, 2002).

Plasmodesmata (PD) are essential but poorly understood structures in plant cell walls that provide symplastic continuity and intercellular communication pathways between adjacent cells and thus play fundamental roles in development and pathogenesis (Lucas, 1994). The nature of the molecular interactions between viral MPs and PD components and their role in viral movement has remained essentially unknown. Here, we show that the family of PD-located proteins (PDLPs) promotes the movement of viruses that use tubule-guided movement by interacting redundantly with tubule-forming MPs within PDs. Genetic disruption of this interaction leads to reduced tubule formation, delayed infection and attenuated symptoms. PDLPs as PD proteins with receptor-like properties involved the assembly of viral MPs into tubules to promote viral movement. The importance of PDLPs in this process was shown when reduction in accumulation led to reduced tubule formation, delayed infection and attenuated symptoms. The PDLPs work together to support virus infection of plants and as such provide important mechanistic insights into the movement mechanism of plant viruses within their hosts (Amari, 2010).

MP modify the plasmodesmata by one of two well-understood molecular mechanisms. The MP of many plant viruses form a transport tubule within the pore of the plasmodesmata that allows the transport of mature virus particles. Examples of viruses that use this mechanism are cowpea mosaic virus (CPMV) and tomato spotted wilt virus (TSWV). The second mechanism by which movement proteins work is by associating with and coating the genome of the virus, causing the ribonucleoprotein complexes to be transported through plasmodesmata into neighbouring cells. TMV's $30 \mathrm{KDa}$ movement protein acts via this mechanism, although it may also have other roles in infection.

The first evidence for a virus-coded MP came from studies with the temperaturesensitive $L s-1$ strain of TMV that is unable to move cell to cell at a restrictive temperature (Heinlein, 2004). This strain can replicate and assemble normally in leaf cells or protoplasts but cannot move cell to cell in leaves at nonpermissive temperature (FaoShan Jing 1978). The defect was correlated with slight changes in the tryptic peptide map prepared from an in vitro translated $30-\mathrm{kDa}$ protein (Joshi, 1983) and was later shown to be due to a single base change in the Ls-1 
genome, which substituted a serine for a proline residue (Ohno 1983). Plants transgenic for TMV $30-\mathrm{kDa}$ protein were shown to complement for Ls-1 at nonpermissive temperatures (Meshi, 1987) and frame-shift mutations in the $30-\mathrm{kDa}$ gene of the virus gave rise to cell-to-cell movement-defective TMV phenotypes.

Since then, this protein has been studied in detail and was reported to bind singlestranded nucleic acids (Citovsky, 1990 and Citovsky, 1992), to accumulate in Pd and to increase their SEL (Dlng, 1992) to localize to the ER and cytoskeletal element, and to be phosphorylated by cellular kinases. The ability of MP to increase Pd SEL was investigated by injection of fluorescencelabelled dextrans into leaf mesophyll cells of transgenic tobacco plants expressing the protein. The Pd in MP-transgenic plants allowed the intercellular diffusion of $10-\mathrm{kDa}$ dextrans, whereas $\mathrm{Pd}$ in non transformed plants restricted the trafficking of dextrans larger than $1.0 \mathrm{kDa}$ (Heinlein, 2004).

\section{Life cycle of plant viruses}

Plant infection with the help of vector, virus inter in to plant cell and uncoat viral genome inside the cytoplasm (Figure 1A\&1B). After disassembly, early transcription and translation lead synthesis of movement protein (MP) (Figure 1Ca) and same time this process, a chamber type structure called replication center assembles in cytoplasm due to the perturbation of internal membranous (Figure 1D).

Replicate and multiplication viral genome in this replication center. After amplification of genome the late transcription and translation lead to the synthesis of $\mathrm{CP}$ and MP as well (Figure 1E\&1F). After protein synthesis, CP recognize signal genomic end and start cocondensing over it, and to form complete virus particle (Figure 1G), compote virus particle is called virion particle or infectious particle. Co- condensation very nicely in potato leaf roll virus (PLRV).

Finally, ultimate goal is complete virus particle (virion) move and spread to the neighbor cells, which is govern by MP (Figure 1H). MP help in either movement of virion particle through enlargement (Figure $1 \mathrm{H})$ or directly genomic material from of nucleoprotein complex to the adjacent cell (Figure 1D, 1Da, 1Db, 1Dc). Finally, complete infectious virus particle exits and transmits to the new host by the help of vector (Figure 1I). Figure 2a,b,c and d represents the genome organization of different plant viruses encoding structural and non-structural proteins (Figure 2a, b, c \& d).

\section{Role of plasmodesmata during viral movement}

Plasmodesmata form long, cylindrical PMlined bridges between neighbouring cells across their cell walls. The cytoplasmic sleeve delimited by the PM within plasmodesmata is occupied by the appressed endoplasmic reticulum (ER) strand and proteinaceous components, which add intricate structural and mechanistic elements to each channel. It is presumed that soluble molecules are transported passively through the cytoplasmic sleeves, which are subdivided into microchannels of 3-4 nm in diameter within the space between the ER and PM (Maule, 2008, Roberts, 2003 and Robards, 1990).

Plasmodesmata can also actively facilitate intercellular trafficking of a special class of endogenous proteins, called non-cell autonomous proteins (NCAPs), and various types of RNA (Lucas, 2004 and Haywood, 2002). Many NCAPs, particularly those that act as transcription factors, have been implicated in developmental processes, such 
as celltype specification and differentiation (Cilia 2004 and Gallagher 2005). Callose (Callose, a $\beta-1,3$ glucan cell wall polymer, is one of the prominent, non-proteinaceous components associated with plasmodesmata. It is reversibly deposited within the cell wall surrounding the neck region of the plasmodesma in response to various stress conditions. Callose deposition is controlled by callose synthases and $\beta-1,3$ glucanases.

Arabidopsis (Arabidopsis thaliana) encodes 12 members of the glucan synthase-like enzyme family that are predicted to function as callose synthases and over 40 glucanases. Reversible control over plasmodesmal permeability would be best achieved by positioning both callose synthase and hydrolase at the plasmodesma. Indeed, a $\beta-1,3$ glucanase has been shown to be targeted to plasmodesmata and one of the callose synthases has been implicated in controlling plasmodesmal trafficking), a $\beta-1,3$ glucan polymer, can be considered a regulatory component of plasmodesmata because its deposition at the plasmodesmal orifice can physically constrict the dimension of the opening, reducing the size exclusion limit (SEL), or even seal it completely, blocking plasmodesmal trafficking (Radford, 1998 and Lee, 2011).

\section{Relationship between virus-induced compartments}

The importance of virus induced membrane compartments as a recurrent theme. Each virus encodes one or more proteins to interact with membranes and to form membraneprotected compartments for virus replication and/ or movement. Since virus movement depends on MPs that facilitate the spread from infected into adjacent cells, MPassociated complexes were originally believed to solely function in the transport of replicated viral genomes from localized VRC compartments to PD for spread. However, as explained in the preceding paragraphs, it now appears that the mobile compartments that target PD are associated with the replication machinery and thus likely contain VRCs themselves.

Thus, the VRC-containing compartments occurring during infection are divided into small, mobile compartments associated with virus movement and larger, sessile compartments that usually occur later and function as viral factories that replicate the virus to produce virion progeny (Grangeon 2012). Viral infection may thus be understood as a rather simple process in which VRCs formed on membranes are either mobile and target PD to spread infection or remain attached and over time grow into factories (Heinlein 2015).

\section{Formation of VRC at the ER}

The formation of ER associated VRCs involves viral proteins with intrinsic affinity to the ER membrane. Since infection starts by association of the viral genome with the ER membrane, as nicely shown for TMV (Christensen, 2009), and the virus-encoded proteins are translated in the vicinity, VRC complexes for transport and/or further replication can be formed.

Although the complexes may be kept together through protein:protein and protein:RNA network interactions, the question arises how these complexes withstand the pulling and pushing forces of a highly fluid membrane loaded with other mobile protein complexes. The formation of distinct replication complexes indicates that the complexes neither dissipate along the network nor occur in endless numbers at random sites. The inhibition of myosin XI-2 led to the dissipation of replicase complexes along the ER thus indicating a role in concentrating 
replicase and other factors at specific ER sites (Amari, 2014 and Heinlein, 2015).

\section{Potential role of aggresomal processes in VRC formation}

Processes of cytoskeleton mediated aggregation of VRCs with host membranes and viral as well as host proteins supports the important role of the cytoskeleton as a scaffold for the anchoring and accumulation of proteins. By providing such anchored accumulation sites the cytoskeleton may support virus infection by increasing the local concentration of viral and host components required for replication, movement, and assembly, and by shielding the virus and its replication from host defense.

Aggregation of proteins with support of cellular scaffolds may also facilitate cellular responses that recognize virus components and target them for storage and degradation. These processes are reminiscent of aggresomal mechanisms in mammalian cells that use microtubules to concentrate protein aggregates at the centrosome for immobilization in inclusions called aggresomes and for their subsequent degradation by proteasomes and/or autophagy (Wileman, 2007).

The similarity of pericentriolar inclusions or "viroplasms" formed by mammalian viruses and aggresomes led to the suggestion that viruses actually subvert aggresomal processes for their life cycle. Viruses may have evolved means to make themselves recognizable as aggregates and thus be targeted to the microtubule-organizing centre (MTOC) to concentrate host and viral proteins in one place to facilitate replication and assembly.

Moreover, in being part of a pathway that delivers proteins for degradation, the viroplasms would have means to facilitate their turnover, thus reducing the toxic effects of accumulated viral proteins once the production of virus progeny is completed. Unlike mammalian cells, plant cells do not concentrate their microtubule nucleating complexes at one MTOC per cell (the centrosome) but rather use them in a dispersed manner throughout the cortical cytoplasm. This may explain why plant viruses usually do not form a single viroplasm per cell but rather multiple VRCs and factories.

As already discussed, the ER-associated MP of TMV may interact with, target or even recruit microtubule-nucleation complexes as centres for cytoskeleton-mediated delivery of host factors to establish VRCs. This phenomenon may suggest that TMV uses its MP to trigger the formation of aggresomes for VRC formation and subsequent degradation. The formation of the VRCs around the MTOCs may be facilitated by the intrinsic ability of the MP to oligomerize (Boutant 2010) and thus to recruit MP-associated ER membranes to microtubules.

The ER provides an elaborate system of protein quality control, which involves chaperones and ubiquitin mediated degradation by proteasomes to reduce protein aggregation (Brandizzi, 2009). Thus, while MP-mediated ER aggregation at the MTOC facilitates the formation of the VRC and viral factories, its accumulation also causes ER stress that subsequently leads to the activation of the adenosine triphosphatase (ATPase) CDC48, which removes MP from the membrane for subsequent ER associated degradation (ERAD) in the cytoplasm (Niehl, 2012 and Niehl, 2013). This phenomenon is likely linked to the unfolded protein response (UPR), a transcriptional program triggered by an increased ER load of misfolded proteins. UPR has been described for several plant viruses and represents an essential mechanism 
to maintain ER homeostasis during infection (Verchot 2014). In the case of TMV, the accumulation of MP and the subsequent induction of these ER homeostasis mechanisms are reflected in the extensive, MP-induced ER membrane remodeling associated with viral factory formation during mid-stages of infection and the subsequent restoration of the normal tubular ER network upon MP clearance during late stages (Heinlein, 1998 and Reichel, 1998).

The balance between the production of MP and its degradation likely represents a major determinant for the size of the ER inclusions. Thus, whereas the high level of MP produced by TMV may saturate the protein degradation system and thus cause its accumulation in the ER and the formation of large inclusions, the MP of ORMV, in contrast, accumulates to low cellular levels and, accordingly, forms only small VRCs (Niehl 2014).It appears remarkable that the MP of ORMV allows for faster virus movement than the MP of TMV despite its lower cellular expression level.

This may indicate that the production of high levels of MP and the formation of larger factories is inhibitory for movement. Higher MP levels leading to MP aggregation may thus reduce the pool of MP available to facilitate the intercellular spread of TMV. However, this hypothesis requires further analysis since a TMV variant that encodes a mutant MP and showed delayed degradation and accumulation in large VRCs was not compromised in its ability to move between cells efficiently (Gillespie, 2002).

While TMV and ORMV may use MTOC as targets for the formation of ER-associated VRCs and derived viral factories, and many other viruses associate with the ER for replication (Bamunu, 2011, Carette, 2000, Grangeon, 2012, Han, 2003, Huang, 1999, Restropo-Hartwig, 1996, Ribeiro, 2008,
Ritzenthaler, 2002, Schaad, 1997, Turner, 2004), other viruses may use different locations to establish their VRCs. For example, Carnation Italian ringspot virus (CIRV) and Melon necrotic spot virus (MNSV) form factories from the outer membrane of mitochondria (Mochizuki, 2009 and Weber-Lotfi, 2002), whereas the tonoplast is implied to play a role in the replication of Alfalfa mosaic virus (AlMV) and Tomato mosaic virus (Hagiwara, 2003, Ibrahim, 2012 and Van Der Heijden, 2001).

\section{MP transport mechanisms}

The ER is linked to the desmotubular ER and thereby provides a direct pathway for the targeting of ER-associated VRCs to PD, mechanisms may play a role. The TGB2 and TGB3 proteins of PMTV associate with endocytic vesicles proposed to be involved in recycling TGB2 and TGB3 back to the ER once they have delivered the VRC to PD. Both proteins were found in association with vesicles stained with FM4-64, a widely used marker of the endocytic pathway.

TGB2 also co localized in vesicles with an Arabidopsis homolog of Rab5 (Ara7), a regulator of vesicle fusions in early endosomes, and interacted with RME8, an endocytic DNAJ chaperone. The MP of TMV and also that of the DNA virus Cabbage leaf curl virus $(\mathrm{CaLCuV})$ interact with the Arabidopsis synaptotagmin SYTA, which regulates endosome recycling. This observation indicates that these MPs reach the PM and that SYTA may support virus movement by allowing these MPs to reach PD through an endosomal recapture pathway.

That the MP of TMV interacts with the PM was recently confirmed by dominant negative inhibition of class VIII myosins, which led to accumulation of MP in the PM rather than at PD, thus indicating that class VIII myosins 
play a role in redirecting the MP from the PM to PD. Since class VIII myosins have been reported to play a role in endocytotic processes, it appears possible that these myosins support a PD-localized endosomal recycling pathway that delivers and retrieves the MP between the ER and the PM at PD and thereby maintains a high concentration of MP at PD. A role of MP associations with the PM in supporting the movement of ER associated VRCs is also indicated by inhibition of PVX movement upon overexpression of Remorin, a protein that interacts with TGB1 and clusters in the PM at PD.

The PD are linked to underlying cortical microtubule:ER junctions/ER-anchoring sites at which VRCs and developing viral factories are formed. Although the ER desmotubule continuum may target VRCs and associated MP from these junctions into adjacent cells, the MP may target PD also by an endosomal pathway that delivers MP to the PM around PD. This pathway may allow MP to interact with the PD-localized and glycosylphosphatidylinositol (GPI)-anchored $\beta$ glucanase AtBG_pap, which is proposed to control callose levels in PD and to play a role in virus movement.

The association of PD with underlying viral factors at microtubule: ER junctions/ER anchoring sites is consistent with the oftenobserved formation of 'paired bodies' or 'caps', thus the accumulation of VRCs and viral proteins on both sides of PD behind the virus front. An association with endosomal compartments also plays a role for the movement of CaMV. Like the already mentioned MP of GFLV, also the MP of this virus (P1) is a "tubule-forming MP", which assembles into tubules within PD and allows the intercellular transport of encapsidated virion particles. P1 carries three Tyr based sorting motifs (YXXФ) that mediate binding to a yet uncharacterized m-like plant adaptin. m2 adaptins are subunits of AP adapter complexes that recognize and recruit cargo proteins into clathrin-coated vesicles. These observations are again consistent with an endocytic pathway that retrieves MPs from the PM and concentrates their localization to the PD. Mutation of the binding motifs in MP abolished its accumulation in PD and its assembly into tubules but not its targeting to the PM, suggesting a role of endosomal trafficking in a pathway that concentrates MP at PD.

The role of endosomal trafficking was confirmed by colocalizing P1 vesicles with the endosomal marker FM6-64 and by inhibition of PD targeting as well as the presence of P1 in FM6-64-tagged vesicles by treatment with Tyrphostin A23, an inhibitor of recognition of Tyr signals by $\mathrm{m} 2$. Tyrbased sorting motifs (YXX $\Phi$ ) were also found in the TGB3 of PMTV and the MP of GFLV and other nepoviruses, thus supporting a widespread role of endoscytic retrieval pathways that direct the MPs from the PM to PD. The MPs of TMV, Turnip vein clearing virus (TVCV) and CaMV were reported to interact with the cell wall protein pectin methyl esterase (PME), which is targeted to the PM by the secretory pathway.

MPs target to plasmodesmata, first plasmodesmal localization signal (PLS) identified in a viral MP, namely, the MP encoded by the Tobamovirus Tobacco mosaic virus (TMV), now provides the opportunity to identify host proteins that recognize this PLS and may be important for its plasmodesmal targeting. One such candidate protein is Arabidopsis synaptotagmin A (SYTA), which is required to form endoplasmic reticulum (ER)-plasma membrane contact sites and regulates the MP-mediated trafficking of poleroviruses, potyviruses, begomo viruses, and tobamo viruses,. SYTA interacts with, and regulates the cell-to-cell 
transport of, both TMV MP and the MP encoded by the Tobamovirus Turnip vein clearing virus (TVCV). Using in planta bimolecular fluorescence complementation (BiFC) and yeast two-hybrid assays, TMV PLS interacted with SYTA. This PLS sequence was both necessary and sufficient for interaction with SYTA, and the plasmodesmal targeting activity of the TMV
PLS was substantially reduced in an Arabidopsis syta knockdown line. SYTA is one host factor that can recognize the TMV PLS and suggest that this interaction may stabilize the association of TMV MP with plasmodesmata (Uan, 2018). MPs mediates cell-to-cell spread of tobacco mosaic virus (TMV) through plant intercellular connections, the plasmodesmata.

Table.1 Viral encoded protein required for cell-to-cell movement of infectious RNA/DNA

\begin{tabular}{|c|c|c|c|c|}
\hline Group & Genus & Virus & MP(s) & Ancillary protein \\
\hline 1 & Polerovirus & PLRV & & $\mathrm{CP}$ \\
\hline 2 & $\begin{array}{l}\text { Tobamovirus } \\
\text { Dianthovirs } \\
\text { Umbravirus }\end{array}$ & $\begin{array}{l}\text { TMV } \\
\text { RCNMV } \\
\text { GRV }\end{array}$ & $\begin{array}{l}30 \mathrm{KDa} \\
35 \mathrm{KDa} \\
\text { ORF4 }\end{array}$ & $\begin{array}{l}- \\
- \\
-\end{array}$ \\
\hline 3 & $\begin{array}{l}\text { Bromovirus } \\
\text { Cucumovirus }\end{array}$ & $\begin{array}{l}\text { CCMV } \\
\text { BMV } \\
\text { CMV }\end{array}$ & $\begin{array}{l}3 \mathrm{a} \\
3 \mathrm{a} \\
3 \mathrm{a}\end{array}$ & $\begin{array}{l}\text { CP } \\
\text { CP }\end{array}$ \\
\hline 4 & Begomovirus & BDMV/SLCV & $\mathrm{BC} 1$ & BV1 \\
\hline 5 & $\begin{array}{l}\text { Potyvirus } \\
\text { Hordei-like viruses }\end{array}$ & $\begin{array}{l}\text { TEV/BCMNV/ } \\
\text { LMV } \\
\text { BSMV }\end{array}$ & $\begin{array}{l}\text { HC-Pro + CP } \\
\text { TGBp1 }\end{array}$ & $\begin{array}{l}\text { CI } \\
\text { TGBp2+ TGBp3 }\end{array}$ \\
\hline 6 & Potex-like viruses & PVX/WCIMV & TGBp1 & TGBp2+ TGBp3 + CP \\
\hline
\end{tabular}

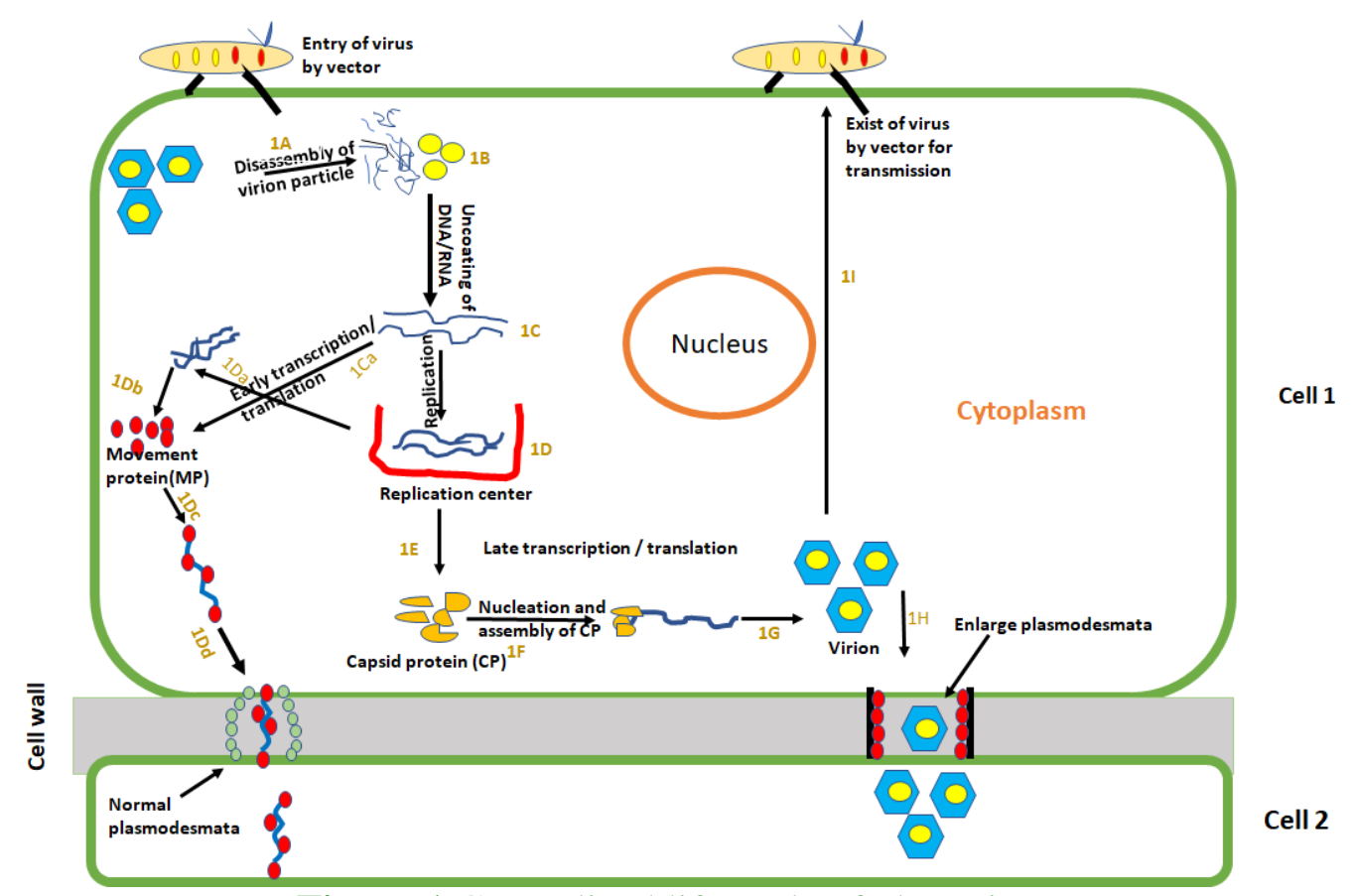

Figure.1 Generalized life cycle of plant viruses 


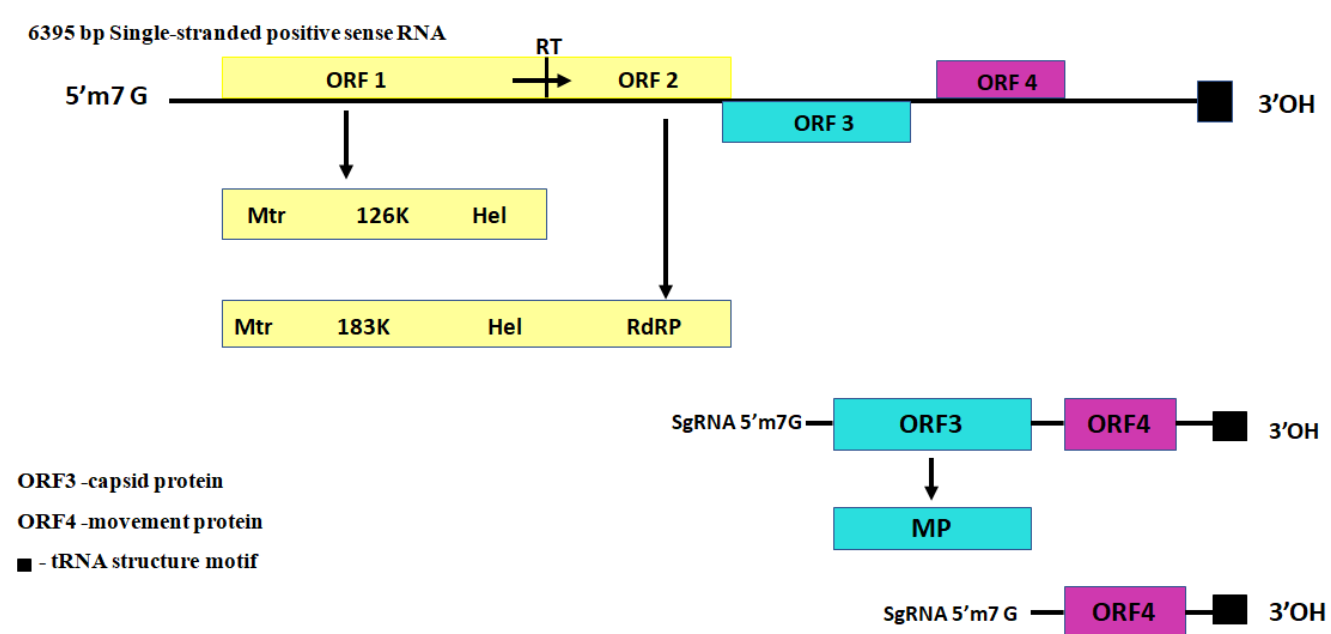

Figure.2a Genome organization of tobacco mosaic virus (TMV)



Figure 2c: Genome organization of Bomovirus

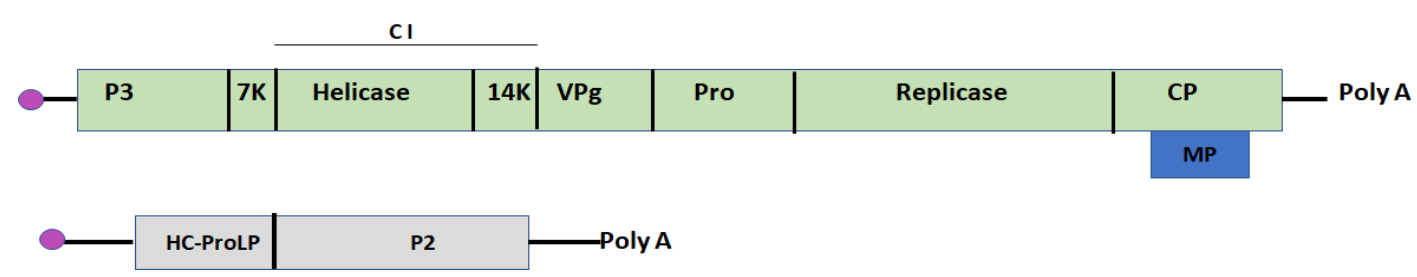

Figure.2b Genome organization of potyvirus



Figure.2c Genome organization of groundnut rosette virus 
- Non-poly-adenylated

- Single-stranded positive sense RNA

- 5883 nucleotides

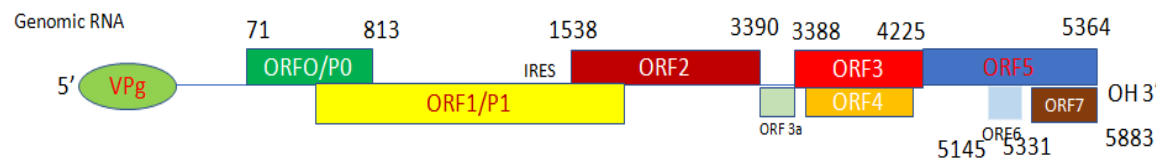

gRNA

- ORF0 -silencing suppressor

- ORF1-polyprotein precursor/protease

- ORF1/ORF2 - Viral RNA-dependent RNA polymerase

- ORF3 -capsid protein

- ORF4 -movement protein

- ORF3/ORF5 -read through transmission protein

- ORF6 -unknown function

- ORF7-PLRV genomic RNA binding protein and

- Rap1-replication-associated protein

3536

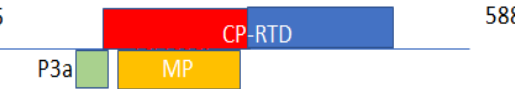

5883 sgRNA1
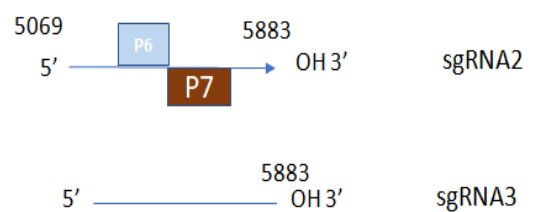

sgRNA3

Figure.2d Genome organization of potato leaf roll virus

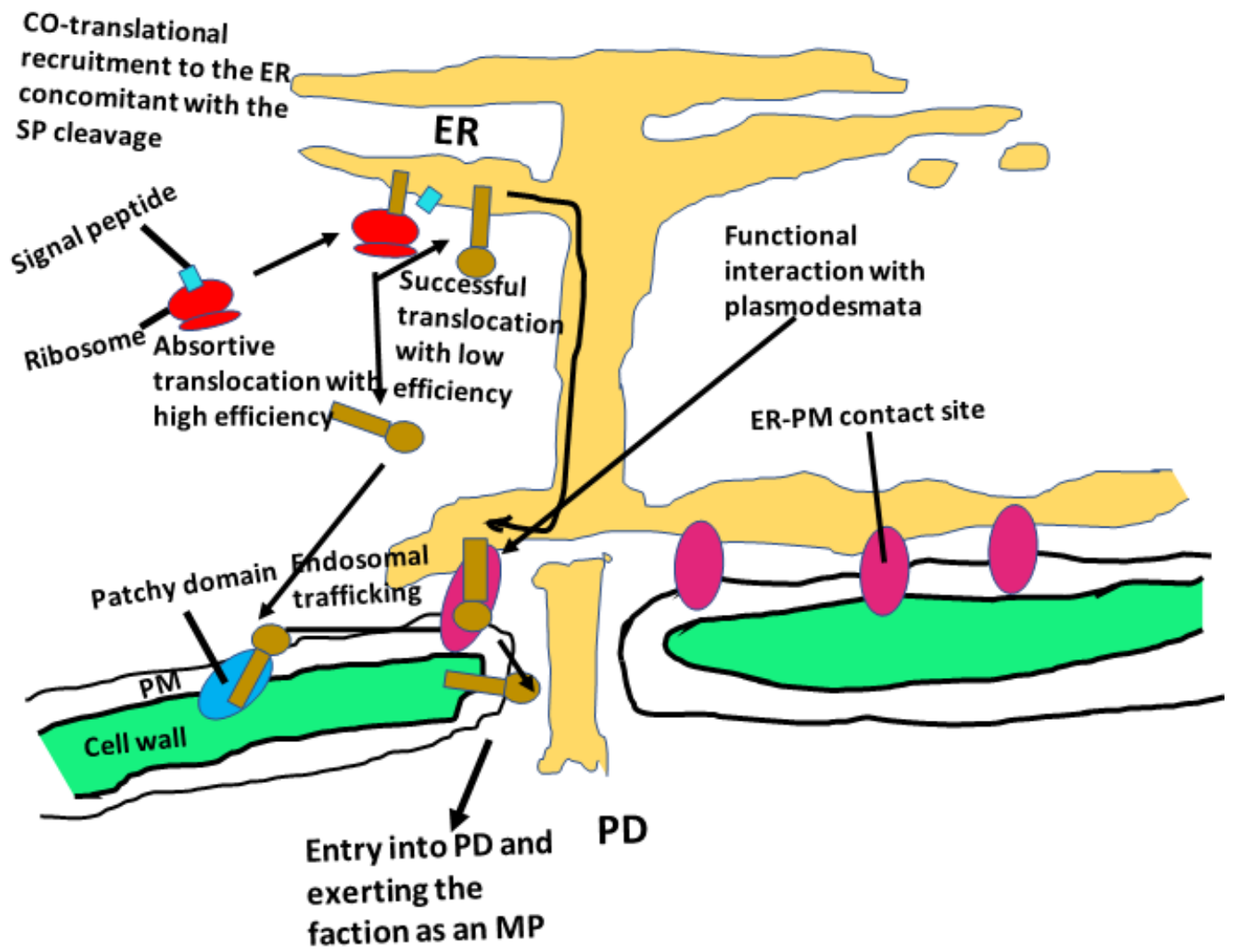

Figure.3 A hypothetical model for the intracellular trafficking of MP 
The molecular pathway by which TMV MP interacts with the host cell is largely unknown. A cell wall-associated protein that specifically binds the viral MP was purified from tobacco leaf cell walls and identified as pectin methylesterase (PME). In addition to TMV MP, PME is recognized by MPs of turnip vein clearing virus (TVCV) and cauliflower mosaic virus (CaMV). The use of amino acid deletion mutants of TMV MP showed that its domain was necessary and sufficient for association with $\operatorname{PME}($ Chen 2000).

Detailed model for the intracellular trafficking of MP has been thoroughly studied by Ishikawa 2017 (Figure 3). MPs use a pathway either through the ER or through the plasma membrane (PM). ER-PM contact sites and PM microdomains, which are subdomains found in the ER and PM, are involved in virus cell-to-cell movement. The intracellular trafficking of fig mosaic virus MP (MP $\left.\mathrm{FMV}_{\mathrm{F}}\right)$ using live cell imaging, focusing on its ERdirecting signal peptide $\left(\mathrm{SP}_{\mathrm{FMV}}\right)$.

Transiently expressed $\mathrm{MP}_{\mathrm{FMV}}$ was distributed predominantly in PD and patchy microdomains of the PM. ER translocation efficiency revealed that $\mathrm{SP}_{\mathrm{FMV}}$ has quite low efficiency compared with SPs of wellcharacterized plant proteins, calreticulin and CLAVATA3. An MP FMV $_{\text {mutant lacking }}$ $\mathrm{SP}_{\mathrm{FMV}}$ localized exclusively to the PM microdomains, whereas SP chimeras, in which the SP of $\mathrm{MP}_{\mathrm{FMV}}$ was replaced by an SP of calreticulin or CLAVATA3, localized exclusively to the nodes of the ER, which was labeled with Arabidopsis synaptotagmin 1, a major component of ER-PM contact sites. low translocation efficiency of $\mathrm{SP}_{\mathrm{FMV}}$ contributes to the generation of ER-translocated and the microdomain-localized populations, both of which are necessary for PD localization. Consistent with this hypothesis, SP-deficient $\mathrm{MP}_{\mathrm{FMV}}$ became localized to PD when co- expressed with an SP chimera. A substantial portion of $\mathrm{MP}_{\mathrm{FMV}}$ that fails to be translocated is transferred to the microdomains, whereas the remainder of $\mathrm{MP}_{\mathrm{FMV}}$ that is successfully translocated into the ER subsequently localizes to ER-PM contact sites and plays an important role in the entry of the microdomain-localized $\mathrm{MP}_{\mathrm{FMV}}$ into $\mathrm{PD}$ (Figure 3) (Ishikawa, 2017).

\section{References}

Amari A, Boutant E, Hofmann C, SchmittKeichinger C, Fernandez-Calvino L, Didier P, Lerich A, Mutterer J R, Thomas C L., Heinlein M, Me Y, Maule A J and Ritzenthaler C. 2010. A Family of Plasmodesmal Proteins with Receptor-Like Properties for Plant Viral Movement Proteins. Volume 6 | Issue 9 | e1001119.

Amari K, Donato M D, Dolja V V and Heinlein M. 2014. Myosins VIII and XI play distinct roles in reproduction and transport of Tobacco mosaic virus. PLoS Pathog 10p: e1004448.

Bamunusinghe D, Seo $\mathrm{J}$ K and Rao A L. 2011.Subcellular localization and rearrangement of endoplasmic reticulum by Brome mosaic virus capsid protein. J Virol, 85: pp. 2953-2963.

Beachy R N and Lucas WJ. 1989. Movement protein of tobacco mosaic virus modifies plasmodesmatal size exclusion limit. Science 246: 377379.

Boevink P and Oparka K J 2005. "Virus-host interactions during movement processes". Plant Physiol 138 (4): 1815-21.

Boutant E, Didier P, Niehl A, Mely Y, Ritzenthaler C and Heinlein M. 2010. Fluorescent protein recruitment assay for demonstration and analysis of in vivo protein interactions in plant 
cells and its application to Tobacco mosaic virus movement protein. Plant J., 62 pp. 171-177.

Brandizzi F, Frigerio L, Howell $\mathrm{S} \mathrm{H}$ and Schafer P. 2014. Endoplasmic reticulum-shape and function in stress translation. Front. Plant Sci 5:p. 425.

Carette J E, Stuiver M, Van Lent J, Wellink J and Van Kammen A. 2000. Cowpea mosaic virus infection induces a massive proliferation of endoplasmic reticulum but not Golgi membranes and is dependent on de novo membrane synthesis. J. Virol74: pp. 6556-6563.

Carluccio A V, Zicca S and Stavolone, L. 2014. Hitching a ride on vesicles: cauliflower mosaic virus movement protein trafficking in the endomembrane system. Plant physiology, 164(3):1261-1270.

Chen M H, Sheng J, Hind G, Handa A K and Citovsky V.2000. Interaction between the tobacco mosaic virus movement protein and host cell pectin methylesterases is required for viral cell- to- cell movement. The EMBO Journal, 19(5): 913-920.

Christensen N, Tilsner J, Bell K, Hammann P, Parton R, Lacomme C and Oparka K. 2009. The 5' cap of Tobacco mosaic virus (TMV) is required for virion attachment to the actin/endoplasmic reticulum network during early infection. Traffic 10: pp. 536-551.

Cilia M L and Jackson D.2004. Plasmodesmata form and function. Curr. Opin.Cell Biol. 16:500- 506.

Citovsky V, Knorr D, Schuste G and Zambryski P. 1990. The P30 movement protein of tobacco mosaic virus is a single-strand nucleic acid binding protein. Cell 60(4): 637-647.

Citovsky V, Wong M L, Shaw A L, Prasad V, B V and Zambryski P.1992.
Visualization and characterization of tobacco mosaic virus movement protein binding to single stranded nucleic acids. Plant Cell 4:397- 411.

Deom C M, Oliver M J and Beachy R N. 1987. The 30-kilodalton gene product of tobacco mosaic virus potentiates virus movement. Science 237:389394.

Desvoyes B N D, Faure-Rabasse S, Chen M H, Park J W and Scholthof H B. 2002. A Novel Plant Homeodomain Protein Interacts in a Functionally Relevant Manner with a Virus Movement Protein. Plant Physiol. Vol. 129.

Dlng B, Haudenshield J S, Hull R J, Wolf S, Roger N, Beachy $b$ and LUCBS J. (1992). Secondary plasmodesmata are specific sites of localization of the tobacco mosaic virus movement protein in transgenic tobacco plants. The Plant Cell, Vol. 4: 915-928.

Fao-Shan Jing M.D., Helmuth Goepfert M.D. and Lanny Garth Close M.D.1978. Computerized Tomography of Paranasal Sinus Neoplasms.Volume88:Issue9, Pages 1485-1503.

Gallagher K L and Benfey P N. 2005. Not just another hole in the wall: understanding intercellular protein trafficking. Genes Dev19:189-195.

Gillespie T., Boevink P., Haupt S., Roberts A.G., TothR.,Valentine T.,Chapman S. and Oparka K.J. (2002). Functional analysis of a DNA-shuffled movement protein reveals that microtubules are dispensable for the cell-to-cell movement of Tobacco mosaic virus.Plant Cell, 14 pp. 1207-1222.

Grangeon R, Agbeci M, Chen J, Grondin G, Zheng $\mathrm{H}$ and Laliberte $\mathrm{J} \mathrm{F}$. 2012. Impact on the endoplasmic reticulum and Golgi apparatus of Turnip mosaic virus infection.J. Virol 86:pp. 9255-9265. 
Grangeon R, Agbeci M, Chen J, Grondin G, Zheng $\mathrm{H}$ and Laliberte $\mathrm{J} \quad \mathrm{F}$. 2012.Impact on the endoplasmic reticulum and Golgi apparatus of Turnip mosaic virus infection

Hagiwara Y, Komoda K, Yamanaka T, Tamai A, Meshi T, Funada R, Tsuchiya T, Naito S. and Ishikawa M.2003. Subcellular localization of host and viral proteins associated with tobamo virus RNA replication. EMBO $J$. 22: 344-353.

Han S and Sanfacon H. 2003. Tomato ringspot virus proteins containing the nucleoside triphosphate binding domain are transmembrane proteins that associate with the endoplasmic reticulum and cofractionate with replication complexes J. Virol.77: pp. 523-534.

Haywood V, Kragler F and Lucas W J. 2002. Plasmodesmata: pathways forprotein and ribonucleoprotein signaling. Plant Cell14: S303-S325.

Haywood V. 2002. Plasmodesmata: pathways for protein andribonucleoprotein signaling. Plant Cell14: S303-S325.

Heinlein M and Epel B L. 2004. Macromolecular Transport and Signaling Through Plasmodesmata. International Review of cyrology .Volume 235, Pages 93-164.

Heinlein M and Epel B L. 2004. Macromolecular Transport and Signaling Through Plasmodesmata. International Review of cyrology .Volume 235: Pages 93-164.

Heinlein M., Padgett H.S., Gens J.S., Pickard B.G., Casper S.J., . Epel B.L. and Beachy R.N. (1998). Changing patterns of localization of the Tobacco mosaic virus movement protein and replicase to the endoplasmic reticulum and microtubules during infection. Plant Cell, 10 pp. 1107-1120.

Heinlein M.2015.Plant virus replication and movement. Virology. Volumes 479480, Pages 657-671.

Huang M and Zhang L. 1999. Association of the movement protein of Alfalfa mosaic virus with the endoplasmic reticulum and its trafficking in epidermal cells of onion bulb scalesMol. Plant Microbe Interact12:pp. 680-690.

Hull R. 1991. The movement of viruses within plants. Semin. Virol. 2:89-95.

Ibrahim A, Hutchens $\mathrm{H} \mathrm{M}$, Berg $\mathrm{R} \mathrm{H}$ and Loesch-Fries L S. 2012. Alfalfa mosaic virus replicase proteins, P1 and P2, localize to the tonoplast in the presence of virus RNA. Virology 449461.

Ishikawa K, Hashimoto M, Yusa A, Koinum H, Kitazawa, Y, Netsu O and Namba S.2017. Dual targeting of a virus movement protein to ER and plasma membrane subdomains is essential for plasmodesmata localization. PLoS pathogens 13(6):e1006463.

J. Virol 86 pp. 9255-9265.

Joshi S, Pleij C W A,Haenni A L,Chapeville F. and Bosch L. 1983.Properties of the tobacco mosaic virus intermediate length RNA-2 and its translation.Virology. Volume127: Issue 1, Pages 100-111.

Jung-Youn Leeand Hua Lu.2011. Plasmodesmata: the battleground against intruders. Trends in Plant Science Vol 16: No. 4.

Lee J Y and Lu H. 2011. Plasmodesmata: the battleground against intruders. Trends in Plant Science. Vol. 16: No. 4.

Lucas W J and Gilbertson R L.1994. Plasmodesmata in relation to viral movement within leaf tissues. Annu. Rev. Phylopalhoi32:387-411.

Lucas W J and Lee J Y. 2004. Plasmodesmata as a supracellular control network in plants. Nat. Rev. Mol. Cell Biol. 5: 712-726. 
Lucas WJ.2006."Plant viral movement proteins: agents for cell-to-cell trafficking of viral genomes". Virology 344 (1): 169-84.

Meshi T, Watanabe Y, Saito T, Sugimoto A, Maeda T and Okada Y.1987. Function of the $30 \mathrm{kd}$ protein of tobacco mosaic virus: involvement in cell- to- cell movement and dispensability for replication. EMBO J. 6:2557-2563.

Mochizuki T, Hirai K, Kanda A, Ohnishi J, Ohki $\mathrm{T}$ and Tsuda S. 2009. Induction of necrosis via mitochondrial targeting of Melon necrotic spot virus replication protein p29 by its second transmembrane domain. Virology 390: 239-249.

Niehl A, Amari K, Gereige D, Brandner K, Mély Y. and Heinlein M. 2012. Control of Tobacco mosaic virus movement protein fate by CELL-DIVISION-CYCLE protein 48 (CDC48) Plant Physiol 160: pp. 20932108.

Niehl A, Amari K. and Heinlein M. 2013. CDC48 function during TMV infection: regulation of virus movement and replication by degradation?

Niehl A.,Pasquier A., Ferriol I., Mély Y. and Heinlein M. (2014).Comparison of the Oilseed rape mosaic virus and Tobacco mosaic virus movement proteins (MP) reveals common and dissimilar MP functions for tobamovirus spread Virology, 456457 pp. 43-54.

Ohno T., Takamatsu N., Meshi T. and Okada Y.1983.Single amino acid substitution in $30 \mathrm{~K}$ protein of TMV defective in virus transport function. Article in Virology 131(1):255-8.

Plant Signal Behav 8 p. e22865.

Radford J E. 1998. Callose deposition at plasmodesmata. Protoplasma201: 3037.
Reichel C. and Beachy R.N. (1998). Tobacco mosaic virus infection induces severe morphological changes of the endoplasmaticreticulum. Proc. Natl. Acad. Sci. USA, 95 pp. 11169-11174.

Restropo-Hartwig M A. and Ahlquist P. 1996. Brome mosaic virus helicase-and polymerase-like proteins colocalize on the endoplasmic reticulum at sites of viral RNA synthesis. J. Virol 70: pp 8908-8916.

Ribeiro D, Forest O, Denecke J, Wellink J, Goldbach R. and Kormelink R J.2008. Tomato spotted wilt virus glycoproteins induce the formation of endoplasmic reticulumand Golgi-derived pleomorphic membrane structures in plant cells. $J$. Gen. Virol 89:1811-1818.

Ritzenthaler C, Laporte C, Gaire F, Dunoyer P, Schmitt C, Duval S, Piéquet A, Loudes A M, Rohfritsch O, stussiGaraud C P and Pfeiffer C. 2002. Grapevine fanleaf virus replication occurs on endoplasmic reticulumderived membranes. J. Virol 76: 88088819.

Robards AWand Lucas WJ. 1990. Plasmodesmata. Annu. Rev.Plant Physiol. Plant Mol. Biol. 41: 369-419.

Roberts AG and Oparka KJ. 2003. Plasmodesmata and the control of symplastic transport. Plant Cell Environ. 26:103-124.

Sanderfoot A A and Lazarowitz S G 1996. Getting it together in plant virus movement: Cooperative interactions between bipartite geminivirus movement proteins. Trends Cell Biol 6: 353-358.

Schaad M C, Jensen P E and Carrington J C. 1997. Formation of plant RNA virus replication complexes on membranes: role of an endoplasmic reticulum-targeted viral protein. EMBO J 16: pp. 4049-4059. 
Sessions A, Yanofsky M F and Weige D. 2000. Cell- cell signaling and movement by the floral transcription factors LEAFY and APETALA1.Science 289:779- 781.

Sondra G Lazarowitz.1999. "Probing plant cell structure and function with viral movement proteins". Current Opinion in Plant Biology 2:332-338.

Turner K A, Sit T.L, Callaway A S, Allen N $\mathrm{S}$ and Lommel S A. 2004. Red clover necrotic mosaic virus replication proteins accumulate at the endoplasmic reticulum. Virology 320: 276-290.

Uan C, Lazarowitz S G and Citovsky V. 2018. The plasmodesmal localization signal of TMV MP is recognized by plant synaptotagmin SYTA. MBio, 9(4):e01314-18.

Van Der Heijden M W, Carette J E, Reinhoud $P$ J, Haegi A and Bol J F. 2001. Alfalfa mosaic virus replicase proteins P1 and P2 interact and colocalize at the vacuolar membrane. J. Virol.75: pp. 1879-1887.

Verchot J. 2014. The ER quality control and ER associated degradation machineries are vital for viral pathogenesis. Front. Plant Sci. 5 p. 66

Waigmann E, Lucas W J, Citovsky V, and
Zambryski P.1994. Direct functional assay for tobacco mosaic virus celltocell movement protein and identification of a domain involved in increasing plasmodesmal permeability. Proc. Natl. Acad. Sci. USA 91: 1433-1437.

Weber-Lotfi F, Dietrich A, Russo $M$ and Rubino L.2002. Mitochondrial targeting and membrane anchoring of a viral replicase in plant and yeast cells. J. Virol 76: 10485-10496.

Wileman T. 2007. Aggresomes and pericentriolar sites of virus assembly: cellular defense or viral design? Аппи. Rev. Microbiol 61:pp. 149-167.

William J Lucas. 2006. Plant viral movement proteins: Agents for cell-to-cell trafficking of genomes. Virology 344:169- 184 .

Wolf S, Deom C M, Maule A J. 2008. Plasmodesmata: structure, function and biogenesis. Curr. Opin. Plant Biol. 11: 680-686.

Wu X L, Dinneny J R, Crawford K M, Rhee Y, Citovsky V, Zambryski P C and Weigel D. 2003. Modes of intercellular transcription factor movement in the Arabidopsis apex. Development 130: 3735-3745.

\section{How to cite this article:}

Priyanka Kumari, Jitesh Kumar, Tushar Ranjan and Ravi Ranjan Kumar. 2020. Movement Protein: A Gatekeeper for Cell-To-Cell Transportation of Plant Viruses. Int.J.Curr.Microbiol.App.Sci. 9(04): 1263-1279. doi: https://doi.org/10.20546/ijcmas.2020.904.149 